\title{
Heuristic methods for solution of FACTS optimization problem in power systems
}

\begin{abstract}
This paper presents a comprehensive review on various heuristic methods for solution of flexible AC transmission systems (FACTS) optimization problem in power systems. First, it classifies FACTS optimization methods into four main groups, then subdivides heuristic methods into different subsets and discusses thoroughly about characteristics, advantages and disadvantages of each heuristic subset. Finally, some hints for future researches on this area will be offered.
\end{abstract}

Keyword: FACTS; Heuristics; Optimization 